\title{
ANÁLISIS DE NECESIDADES EDUCATIVAS CURRICULARES EN LA EDUCACIÓN PRIMARIA DIRIGIDA A NIÑOS(AS) NICARAGÜENSES MIGRANTES EN COSTA RICA
}

\author{
Nidia Esperanza Mejía Arias ${ }^{1}$ \\ Docente en la Universidad Nacional BICU de la Costa Caribe de Nicaragua \\ Nicaragua
}

Recibido 17 de junio 2007 • Aprobado 20 de agosto 2007

\begin{abstract}
Resumen: Con este artículo se pretende dar a conocer las necesidades curriculares identificadas en niñas y niños nicaragüenses migrantes que estudian en la escuela primaria rural Los Ángeles del Cantón Los Chiles de Costa Rica. Ellos y ellas, actualmente, se encuentran en una situación de exclusión, discriminación e indiferencia por parte de las instituciones locales. Existen obstáculos para el aprendizaje efectivo y el desarrollo de actitudes positivas, entre ellos la falta de participación responsable de la comunidad educativa, el procedimiento metodológico utilizado en el aula y la adecuación curricular del modelo pedagógico oficial costarricense; divorciados éstos, de las condiciones socioculturales de esta población educativa, de su familia y de la comunidad rural donde residen.

Esta situación afecta la motivación de las niñas y niños, quienes no alcanzan las metas educativas: la mayoría de los que se matriculan dejan inconclusa la educación primaria, y los que la completan tienen dificultades académicas para ingresar a secundaria.

Los sistemas educativos de Nicaragua y de Costa Rica deben plantear estrategias educativas conjuntas, que den respuesta a las necesidades educativas curriculares que presentan los niños nicaragüenses migrantes en las escuelas rurales costarricenses.
\end{abstract}

Palabras clave: Niños migrantes, perfil de las poblaciones inmigrantes, necesidades curriculares de educación de los niños y las niñas migrantes.

Abstract: The intention of the article is to announce the needs of a curriculum Identified in the Nicaraguan migrant children that study at the rural primary school "Los Angeles" in the location of Los Chiles Costa Rica. They actually are in an exclusion, discrimination and indifference situation from the local institutions. There are strong obstacles for the effective learning and development of positive attitudes, lack of responsible participation of the educative community; the methodological procedure used at the classrooms and the curricular adjustment at the pedagogical official model from Costa Rica, divorced those, from the sociocultural conditions of the children, their families and the rural community where they live.

Maestra de Educación Primaria, Especialista en Educación de Adultos, Maestra en Planeación y Desarrollo/Investigadora profesional/ Docente en la Universidad Nacional BICU de la Costa Caribe de Nicaragua; Especialista Curricular del MINED de Nicaragua/. Miembro activo de la Red de Educación Rural y de Formadores de Docentes Centroamericanos. Correo electrónico: mejianidia9@hotmail.com 
This situation affects the children motivation, for not achieve educational goals, the few that register at primary school doesn't end it and those that complete it have academic difficulties to enter at high school.

The time has come for the educational systems from both countries -Nicaragua and Costa Rica- to consider joint educational strategies that can give an answer to the educational curricular needs that the Nicaraguan migrant children presents at the rural schools in Costa Rica.

Key words: Migrant children, migrant population profile, curricular needs of education for migrant children.

\section{INTRODUCCIÓN}

La migración de las personas se debe a muchos factores, de ellos, el más fuerte, es la búsqueda del bienestar personal y familiar. Este fenómeno es histórico, pues cuando la persona o la familia ha agotado los esfuerzos por lograr reconstruir una vida satisfactoria, emigra por un sueño que no siempre se hace realidad.

La migración nicaragüense hacia Costa Rica se intensificó en la década de los 90 por motivos bélicos y desastres naturales; después, la falta de empleo intensificó las dificultades económicas en la familia nicaragüense, lo que provocó una masiva migración; de manera que, en el 2005, se registró una población migrante de, aproximadamente, unas 600000 personas hacia Estados Unidos y Costa Rica, según French (2005). Cuatro de cada diez migrantes son niños y niñas, quienes son afectados ante los retos de una nueva e incierta vida.

Los Chiles, cantón de Costa Rica, al cual pertenece la escuela rural Los Ángeles, sede de la investigación, cuenta con una matrícula de 97 estudiantes según su registro (Corea, 2006). El Cantón es considerado como uno de los sectores que muestran mayor vulnerabilidad y rezago en lo que respecta a educación, y registra el más alto número de niños nicaragüenses migrantes en la educación primaria. Esta realidad provoca mayor demanda de atención integral: salud, educación, vivienda y trabajo; necesidades básicas que están insatisfechas y sin garantías sociales, afirman los estudios de Costa Rica (Costa Rica, Ministerio de Planificación Nacional y Política Económica [MIDEPLAN], 2005).

En lo que respecta a la educación de los migrantes, se indican bajos niveles de escolaridad en relación con el conjunto de la población costarricense. Esto representa una problemática nacional, porque la educación va de acuerdo con la calidad de vida de la población: Si bien las deficiencias en el acceso a la educación se presentan en todos los grupos etarios: la población preescolar, los adolescentes y los jóvenes, son los que más sufren de la exclusión del sistema educativo costarricense, en tanto son incorporados a la fuerza laboral (Comisión para la Defensa de los Derechos Humanos en Centroamérica [CODEHUCA], 2005). Según el Ministerio de Educación de Costa Rica (MEP, 2005), se tienen 34046 estudiantes nicaragüenses, y el país no posee una política migratoria integral ni una política curricular específica que atienda este tipo de escolaridad.

La asistencia general a la educación en las escuelas costarricenses presenta una brecha muy significativa entre la población escolar costarricense y la nicaragüense migrante, por ejemplo: de los niños de cinco a 6 años que asisten a la escuela, el 44,5\% son inmigrantes nicaragüenses y el 65,3\% son costarricenses (Castro, 2002). Es de reflexionar que si todos los niños nicaragüenses inmigrantes lograran su ingreso y promoción a la educación inicial y 
básica, las probabilidades de superar sus condiciones de pobreza serían más altas, resultado que también beneficiaría a la sociedad costarricense, pues es de consenso que la educación mejora la vida de las personas.

La situación de los niños en condiciones de alta vulnerabilidad, motiva a cualquier investigador a reflexionar sobre la problemática existente, a conocer la realidad de cerca, principalmente en lo referente a las insatisfacciones de las necesidades educativas básicas del niño como ser humano que ha tenido que emigrar por razones que todavía no logra comprender; pero con la esperanza de satisfacerlas, encontrar equidad social, recibir afecto y sentirse seguro, protegido ante la vulnerabilidad de sus derechos como niño o niña migrante, entre otros.

Por ello, se realizó un estudio en la escuela rural Los Ángeles de los Chiles, para conocer las condiciones sociales de la niñez inmigrante nicaragüense que estudia en el nivel de primaria; con el fin de identificar los factores que afectan el aprendizaje, la permanencia en la escuela, la promoción y la continuidad en su educación. Este conocimiento será un insumo para plantear estrategias metodológicas que mejoren la participación comunitaria, la atención a la diversidad cultural y el desarrollo socio afectivo en beneficio de los niños y las niñas.

El estudio consta de dos partes: el análisis y el proyecto. El análisis de las necesidades curriculares de los niños nicaragüenses migrantes que estudian en la escuela Los Ángeles de los Chile, involucró una población muy diversa pero relacionada con la educación y la vida de la escuela: padres de familia, maestros, estudiantes, docentes de la escuela, organizaciones de la comunidad, autoridades educativas locales, docentes de otras escuelas primarias relacionados con este centro educativo, e investigadores profesionales sobre la temática, cuyos criterios y opiniones sobre los hechos fueron la base fundamental del análisis.

La información que se presenta en la primera parte se obtuvo mediante técnicas y métodos variados que facilitaron la interpretación de los hechos, los pensamientos, las expresiones, los significados, los sentimientos y todo aquello que da sentido a la participación de los actores en el proceso educativo de las niñas y niños: la observación participativa en las aulas de clase y de la vida académica de los estudiantes, la entrevista no estructurada con diferentes actores, la historia de vida de una muestra de estudiantes de los últimos grados de primaria, el diario de campo con datos del recorrido por la comunidad, las fotografías del entorno, la observación documental de la historia de la escuela y su evolución, la visita a los hogares de los niños y de algunos líderes comunitarios.

Los resultados del análisis sirvieron de base para la segunda parte del proceso investigativo: el diseño del proyecto que consiste en una propuesta de guía metodológica que contribuya a dar respuesta a las necesidades curriculares que presentan los niños nicaragüenses migrantes. La metodología para elaborar el proyecto en la fase de ejecución se basa en los pasos del marco lógico, su monitoreo y el proceso de evaluación. El consenso, la negociación y la participación entre los diferentes actores, son métodos de trabajo para que la propuesta sea aceptada y sus resultados se incorporen a la vida académica de la escuela.

Se analizaron importantes fuentes bibliográficas con tópicos variados, pero relacionados con el tema en estudio: el tipo de educación que reciben los niños en las escuelas primarias de Costa Rica, los estudios sobre las condiciones de desigualdad en las que trabajan sus padres, el análisis de la actitud discriminatoria de algunos sectores de la sociedad costarricense hacia los nicaragüenses, los acuerdos y los compromisos de los gobiernos centroamericanos acerca de la protección de la mujer y los derechos del niño, así como las críticas de la sociedad civil para los decisorios por la lenta formulación de leyes, acuerdos y políticas, que mejoren la situación de los migrantes nicaragüenses y, fundamentalmente, la de niños y jóvenes que conforman la población más vulnerable. 


\section{PRESENTACIÓN DE LOS DATOS}

La forma de vida y costumbres del ciudadano de los Chiles de Costa Rica es muy similar a la vida en las ciudades nicaragüenses, mas no así en las comunidades rurales. En los sectores rurales de Nicaragua se encuentra poco incentivo para el trabajo, lo que hace que la vida campesina sea difícil. Igual suerte se vive en los campos rurales de Costa Rica; si bien es cierto, los migrantes encuentran trabajo, tienen que hacerlo muy duro para hacer frente a los gastos de la familia. Los nicaragüenses radicados en Los Chiles describen esta localidad como un lugar que ha sido ruta histórica de los nicaragüenses y costarricenses para el comercio del látex y del ganado, fundamentalmente. Cuenta con un área total de 1358 kilómetros cuadrados y 11367 habitantes, está ubicada en las llanuras de los guatusos de la región norte de Costa Rica limítrofe con Nicaragua (Corea, 2006).

La escuela Los Ángeles fue diseñada para ser una escuela regular y hoy, (2007) es una escuela multigrado en la que estudian los niños nicaragüenses migrantes. Los componentes estructurales físicos de la escuela están en buen estado; sin embargo, el equipamiento didáctico es mínimo y, además, inadecuado para la función que debe desempeñar una escuela multigrado, con alta diversidad cultural y donde la mayoría de los estudiantes son migrantes.

\section{Tabla $\mathrm{N}^{\mathrm{o}} 1$}

Componentes estructurales de la escuela Los Ángeles en 2007

\begin{tabular}{lcccc|}
\hline Componentes & Total & Buen estado & Regular & Malo \\
\hline Aulas & 3 & - & $\mathrm{X}$ & - \\
Comedor & 1 & $\mathrm{X}$ & - & - \\
Servicios sanitarios & 3 & $\mathrm{X}$ & - & - \\
Pilas & 3 & $\mathrm{X}$ & - & - \\
Bodega & 1 & - & $\mathrm{X}$ & $\mathrm{X}$ \\
Sala Administrativa & 1 & - & - & \\
Área de juego & 1 & & & \\
\hline
\end{tabular}

Fuente: Visita a la Escuela Los Ángeles en febrero de 2007.

Los pupitres de las aulas de la escuela son iguales, son utilizados por niños de diferentes edades y tamaños, desde preescolar hasta sexto grado, situación que se torna incómoda, para los niños menores. El equipamiento didáctico lo constituye una pizarra; se carece de biblioteca, de juegos didácticos y deportivos, de guías para los estudiantes, de murales y de materiales que les permitan a los niños trabajar de acuerdo con un enfoque metodológico apropiado para el aula multigrado. Las paredes y los asientos están desteñidos, viejos y, en su mayoría, deteriorados.

El entorno a la escuela es un potrero muy húmedo donde pastorean animales. En las horas de receso o juego, los niños se van a sus casas, ubicadas en el vecindario, porque no tienen organizado 
el descanso y, según los docentes, porque hacen falta materiales recreativos, de apoyo al aprendizaje, para la educación física, juegos de mesa y otros. Se pierden minutos en el horario escolar diario en el ir y venir de los niños, situación que propicia peleas y discusiones entre ellos.

Por otro lado, la matrícula se realiza rutinariamente durante tres días. Se obvian las visitas familiares, pues se espera que el padre de familia lleve a su hijo(a) voluntariamente a la escuela, y se carece de alternativas sobre qué hacer cuando los niños no llegan a la escuela. Generalmente, muchos niños no asisten a clases; se carece de estrategias para hacerlos llegar y se desconoce cuál es la demanda escolar en el sector de la escuela.

Tabla $\mathrm{N}^{\mathrm{o}} 2$

Matrícula 2007 de la Escuela Los Ángeles

\begin{tabular}{|cccc|}
\hline Nivel & Varones & Mujeres & Total \\
\hline Transición & - & -2 & 5 \\
Materno & - & - & 10 \\
I & 9 & 12 & 21 \\
II & 8 & 3 & 11 \\
III & 8 & 3 & 11 \\
IV & 9 & 5 & 14 \\
V & 5 & 5 & 10 \\
VI & 7 & 3 & 92 \\
\hline TOTAL & 46 & 31 & 92 \\
\hline
\end{tabular}

Fuente: Dirección Escuela Los Ángeles, febrero de 2007.

${ }^{2}$ No suministraron el dato por género.

De los 92 estudiantes (100\%) matriculados, solo 18 son costarricenses, los demás son nicaragüenses. Los tres docentes son costarricenses y tienen limitaciones para desarrollar las clases, porque no son especialistas en metodologías para multigrado, han aprendido con la práctica, y no reciben capacitación previa en esta modalidad para niños migrantes. Se dan pocas visitas de seguimiento para apoyar la labor de los docentes, y cuando se supervisa es para verificar la asistencia, la matrícula y otros aspectos administrativos. Esto lo expresan los docentes y, lo reconocen los técnicos del Ministerio, quienes aducen que se carece de recursos para visitar las escuelas urbanas y, mucho menos, las rurales. Agregan, que ellos también carecen de la capacitación adecuada para orientar una educación inclusiva.

La propuesta pedagógica de la escuela Los Ángeles no tiene relación con el contexto comunitario ni con lo que sucede en los diferentes escenarios que afectan la educación; no se realizan diagnósticos, se carece de la participación de los padres de familia y representantes de instituciones locales en la planificación educativa de la escuela. Los docentes ignoran las necesidades de los niños en su condición de migrantes; algunos, se ven forzados por sus padres a trabajar para ayudar a la subsistencia familiar, y no existen programas que los protejan en su derecho a la educación. La máxima preocupación de los docentes, es el cumplimiento del programa oficial, el que, además, 
se constituye para ellos en la única fuente de donde se nutre la enseñanza y el aprendizaje de los estudiantes.

La actitud de los niños y las niñas es de desmotivación; expresan que tienen pocas esperanzas de ingresar en la universidad, dicen que eso es como un sueño, porque es difícil aprobar los exámenes. Así lo indican los resultados de su educación primaria en los últimos años.

El número de estudiantes que llegan al sexto grado es muy bajo en relación con el ingreso de primer grado. En el 2004, la matrícula de sexto grado fue sólo de tres estudiantes; en el 2006 llegó a 6, y, en el 2007 se matricularon 7. A pesar de ser tan pocos los alumnos de sexto grado, no logran graduarse: se registra que en el 2006, sólo tres estudiantes de sexto grado aprobaron (50\%).

\section{Tabla $\mathrm{N}^{\mathrm{o}} 3$}

Situación académica de los estudiantes-2004

\begin{tabular}{ccccc|}
\hline Matrícula & Deserción & Repitientes & Aprobados & Aplazados \\
\hline 79 & 9 & 8 & 42 & 20 \\
\hline
\end{tabular}

Fuente: Dirección de la Escuela Los Ángeles.

El número de aplazados es muy alto, como se puede observar en la Tabla $\mathrm{N}^{\mathrm{o}}$ 3: de un total de 79 estudiantes matriculados, se aplazaron 20 en el 2004. Los docentes explican que se aplazan porque pierden los exámenes estandarizados del MEP. La evaluación es estándar, como si la atención y las condiciones fueran homogéneas para las zonas urbana y rural. La realidad es otra, pues las escuelas urbanas gozan de mejores condiciones de trabajo, más control de la asistencia y del aprendizaje de los estudiantes. La aplicación del sistema evaluativo perjudica a los estudiantes de las escuelas rurales, generando situación de inequidad y de exclusión, por lo que no es posible que los(as) estudiantes completen su educación básica.

Algunos niños de la escuela Los Ángeles expresaron, por escrito, parte de las preocupaciones y esperanzas que en ellos se albergan:

Mi nombre es Elvin, estoy en sexto grado, tengo 16 años y 10 de vivir en Costa Rica. Me gustaría volver a mi país, conocer las escuelas, los uniformes, observar las clases, saber si las mismas clases que dan aquí se dan en Nicaragua. Lo que más desearía es volver a ver a mi abuelito, pues tengo muchos años de no verlo, abrazarlo y decirle cuánto lo quiero, que me cuente de nuevo recuerdos de cuando yo era un bebé de cinco años. Me gustaría que vinieran profesores de Nicaragua para observar las enseñanzas que dan aquí.

Me llamo Diana, tengo 10 años de vivir en Costa Rica. Uno de mis mayores deseos es ir a Nicaragua, para conocer las escuelas, los colegios, cómo hablan, como viven, qué clase de trabajo hacen. Quiero ver a mis abuelos, tíos, primos, conocer los parajes, los ríos, las casas, la gente; conversar con otros niños y preguntarles qué hacen en el campo, con el arroz, los frijoles y otras cosechas. Me imagino que Nicaragua debe verse bonita, alegre. Los lugares que más me gustaría conocer son Juigalpa, Managua y San Carlos.

Me llamo Belquin Tamara, estoy en quinto grado, y deseo regresar a Nicaragua, a mi país donde nací, donde tengo a toda mi familia: a mi papá, a mis hermanos y a otros familiares. Deseo cumplir mis 
sueños de regresar, definitivamente, a mi Nicaragua, porque soy nicaragüense por gracia de Dios. Quiero ver a los maestros nicaragüenses.

Mi nombre es Yaritsa, estoy en quinto grado, tengo 12 años y 10 de estar en Costa Rica. Me gustaría conocer Nicaragua, debe de ser muy linda. Desde que vine nos instalamos en Los Hernández, llegué muy pequeña, cuando hago recuerdos me pongo triste, mi papá nos abandonó, pero mi mamá encontró a otro hombre y la pasamos mejor.

Me llamo Fabiola, tengo 15 años y 10 de vivir en Costa Rica, hace tanto tiempo que dejé Nicaragua que ya no la conozco, ahí dejé a mis abuelos, tíos y tengo tantas ganas de conocerlos, así como, también, las escuelas y cómo es la educación. Deseo andar por todos esos lugares.

Me llamo Javier, tengo 12 años y 10 de vivir en Costa Rica, quiero conocer Nicaragua, llegar por agua, saber cómo son los niños, sus uniformes, las escuelas, recibir clase, tener compañeros, llegar hasta Chichigalpa.

Añoranzas y sueños se juntan a la vez.

\section{DISCUSIÓN}

El factor común de la emigración de Nicaragua es la pobreza. La situación en Costa Rica no mejora para los inmigrantes, porque parte de sus ingresos es enviado a la familia que dejaron en su país, nada cambia en sus vidas; la pobreza sigue siendo el mayor problema que enfrentan las familias y, especialmente, los niños nicaragüenses inmigrantes que estudian en las escuelas rurales costarricenses. Los padres de familia ven con incertidumbre el porvenir de sus hijos; pues han perdido la esperanza y la confianza de que Nicaragua se recupere pronto para que haya fuentes de trabajo y poder regresar nuevamente a su patria.

Los trabajos que realizan los migrantes en Costa Rica son los más pesados y con la menor paga, por ejemplo: cortar caña bajo un sol inclemente o recoger café con bajas temperaturas y sin protección, vender diferentes artículos en la calle, ser empleada doméstica con una paga inferior a lo estipulado por la ley; hacer cualquier cosa por unos cuantos colones; y, sobre todo, soportar con estoicismo el trato despectivo: "Ya parecés nica", "Acaso sos nica". El significado del gentilicio "nica" se ha generalizado para hombre o mujer, niño o adulto, y, por lo general, es usado en Costa Rica para comparar, molestar o burlarse de la persona a quien se dirige.

El currículo de educación nacional costarricense necesita ser repensado en una nueva propuesta que tome en cuenta la situación de la niñez nacional y la realidad que viven a diario los niños migrantes: en su mayoría, éstos trabajan después de las clases, en el hogar, en las calles, en el campo y, a veces, abandonan, por períodos, la escuela; porque para ellos, lo primero es el trabajo, de ello depende su sobrevivencia, afirma la familia.

Un currículo flexible y pertinente debería tomar en cuenta la valiosa experiencia que poseen los beneficiarios, en este caso los niños nicaragüenses migrantes, cuyas habilidades, destrezas y valores forjados en las experiencias del mundo del trabajo, incorporados al currículo, les serán útiles en el aprendizaje escolarizado.

La niñez migrante tiene derecho a recibir una educación en condiciones de igualdad de oportunidades y a permanecer en la escuela, a ser alentados, motivados y asistidos en la preservación de su identidad cultural, a desarrollar los valores nacionales de su país de origen, 
a ser protegida para que no interrumpa su educación, a desarrollar su personalidad con dignidad, y a gozar de todos los beneficios que brinda la legislación costarricense para los niños y las niñas. La educación debe ser de calidad, pertinente y significativa, no debe ser discriminatoria ni contraria a los derechos humanos universales suscritos por los países centroamericanos.

Es necesario establecer mecanismos que aseguren la educación de todos, y se hagan efectivos los derechos del niño, reconocidos en la Convención Internacional (UNICEF, 1989) y suscritos en los Códigos Nacionales.

\section{CONCLUSIONES}

1. Los niños y las niñas nicaragüenses migrantes que estudian la educación primaria en escuelas rurales costarricenses, presentan necesidades educativas curriculares, las que hacen que su aprendizaje no sea tan efectivo, entre ellas: a) los educadores con debilidades en los métodos de enseñanza, b) una enseñanza rutinaria y directiva, c) serias dificultades para aprovechar la diversidad cultural de los niños, d) una adecuación curricular que obvia el perfil característico del(a) niño(a) migrante, e) una educación sin participación de la familia y de la comunidad,f) una formación desvinculada de las realidades del fenómeno de la inmigración, g) una educación elitista, ya que son pocos los niños nicaragüenses inmigrantes que ingresan en la escuela, y a los que se les garantiza su permanencia.

2. El rendimiento académico de los estudiantes, a medida que avanzan en la educación primaria, decrece; a tal grado que, de 79 matriculados en primer grado, en la escuela objeto de este estudio, sólo pueden completar la primaria tres ó cuatro estudiantes. Los niños opinan que la escuela es aburrida, pues no se cuenta con las condiciones: materiales de recreación y de libertad, juego, deportes, música, pintura, dibujo y fomento de la creatividad, pues la educación está centrada en el aprendizaje de algunos aspectos de contenido disciplinar de los programas. Esto se deriva de la aplicación de un currículum educativo formal nacional, el cual, desde su concepción, se aparta de la realidad existente en el entorno: condiciones sociales, culturales, psicológicas y económicas de la población migrante, la cual representa el 7,8\% de la población total.

3. La formación de los docentes carece de una preparación en psicopedagogía, con la cual se pueda entender y atender la diversidad de los niños migrantes en todas sus dimensiones, para ayudarlos a construir una vida armoniosa en la cultura costarricense, sin renunciar a su identidad. La metodología y la contextualización curricular que se aplican son inadecuadas tanto para los niños y las niñas inmigrantes, como para su familia: no se motivan la permanencia en la escuela, el aprender a aprender y el continuar aprendiendo después de la educación primaria. Los(as) docentes tienen limitaciones para superar la diferencia cultural, la cual se ha estigmatizado entre los niños nicaragüenses y costarricenses, lo que genera discriminación, lo que entorpece la posibilidad de un aprendizaje armónico de la niña y el niño, y se limita así su adaptación a la cultura costarricense. 


\section{REFERENCIAS}

Castro, C. (2002). Migración nicaragüense en Costa Rica: población, empleo y necesidades básicas insatisfechas. Costa Rica: Facultad Latinoamericana de Ciencias Sociales.

Comisión paara la Defensa de los Derechos Humanos en Centroamérica [CODEHUCA]. (2005). Situación de la población migrante nicaragüense en Costa Rica. San José, Costa Rica.

Corea, A. (2006). Centro educativo Los Ángeles. Alajuela, Costa Rica: Dirección Regional de San Carlos, Los Chiles.

Costa Rica. Ministerio de Planificación Nacional y Política Económica [MIDEPLAN]. (2005). Plan Nacional de Desarrollo 2002-2006. Las iniciativas relativas al acceso a la educación y a la formación. San José: Autor.

Fondo de las Naciones Unidas para la Infancia UNICEF' (1989, noviembre). Convención sobre los Derechos del Niño. UNICEF.

French, A. (2005). Informe: Los Derechos del niño en Nicaragua. Secretariado General-Oficina de Asuntos Internacionales y Derechos Humanos. Consultado el 10 de juniode de: http:/www. ochchr.org/english/bodies/crc/cres39.htm Ministerio de Educación de Costa Rica (2005). Oficina de Estadística. San José, Costa Rica: Departamento de Estadística-MEP. 